\title{
Perfil de multimorbidade associado à incapacidade entre idosos residentes na Região Metropolitana de Belo Horizonte, Brasil
}

\author{
Multimorbidity profile associated with disability among the elderly \\ living in the Metropolitan Region of Belo Horizonte, Brazil
}

Gabriella Marques Bernardes (https://orcid.org/0000-0003-0042-3364) ${ }^{1}$ Juliana Vaz de Melo Mambrini (https://orcid.org/0000-0002-0420-3062) 1,2 Maria Fernanda Lima-Costa (https://orcid.org/0000-0002-3474-2980) 1,2 Sérgio Viana Peixoto (https://orcid.org/0000-0001-9431-2280) 1,2,3
${ }^{1}$ Programa de PósGraduação em Saúde Coletiva, Instituto René Rachou, Fiocruz. Av. Augusto de Lima 1715, Barro Preto. 30190-009 Belo Horizonte Brasil. sergio.peixoto@fiocruz.br ${ }^{2}$ Instituto René Rachou, Fiocruz. Belo Horizonte MG Brasil.

${ }^{3}$ Escola de Enfermagem, Universidade Federal de Minas Gerais. Belo Horizonte MG Brasil.
Abstract The scope of this paper was to investigate the associations of disability in three domains (BADL, IADL and mobility) with cardiovascular diseases, diabetes and multimorbidity profile, among the elderly living in the Metropolitan Region of Belo Horizonte (MRBH). A cross-sectional study was conducted with a representative sample of 2,172 elderly persons (60 years and over). Disability, for each domain (BADL, IADL and mobility), was assessed as reporting great difficulty or need for help to perform at least one activity among those investigated, and self-reported diseases included arterial hypertension, myocardial infarction or angina, stroke, diabetes, and combinations of these diseases. Adjusted Poisson regression was used, and the attributable population fraction was also estimated. A major contribution of Cerebral Vascular Accidents (strokes) to disability in all domains was observed, especially BADLs, as well as the presence of infarction or angina in disability in IADLs and mobility, especially when combined with diabetes and hypertension. The multimorbidity profile can be used to identify vulnerable groups, which should be the target of prevention and rehabilitation, reducing the financial and social cost of this event among the elderly. Key words Disability, Elderly persons, Chronic illness, Morbidity surveys, Cardiovascular diseases
Resumo $O$ objetivo foi investigar as associações da incapacidade em três níveis ( $A B V D, A I V D$ e mobilidade) com doenças cardiovasculares, diabetes e multimorbidade, entre idosos residentes na Região Metropolitana de Belo Horizonte $(R M B H)$. Estudo transversal em amostra representativa de adultos da RMBH, tendo sido selecionados 2.172 idosos (60 anos ou mais). A incapacidade, para cada domínio (ABVD, AIVD e mobilidade), foi avaliada como o relato de muita dificuldade ou necessidade de ajuda para realizar pelo menos uma atividade entre as investigadas, $e$ as doenças autorreferidas incluíram hipertensão arterial, infarto do miocárdio e angina, acidente vascular encefálico (AVE), diabetes, e as combinações dessas doenças. Utilizou-se regressão de Poisson ajustada, estimando-se também a fração atribuivel populacional. Houve importante contribuição do AVE para a incapacidade em todos os domínios, com destaque para as $A B V D$, bem como do infarto/angina na incapacidade em AIVD e mobilidade, sobretudo quando combinadas com diabetes e hipertensão. O perfil de multimorbidade pode ser usado para identificação de grupos vulneráveis, que deveriam ser alvo de ações de prevenção e reabilitação, reduzindo o custo financeiro e social desse evento entre idosos.

Palavras-chave Incapacidade, Idoso, Doença crônica, Inquéritos de morbidade, Doenças cardiovasculares 


\section{Introdução}

As mudanças nos perfis demográfico e epidemiológico das populações levam ao aumento das doenças crônicas não transmissíveis, que aparecem frequentemente associadas à incapacidade para realização de atividades diárias e o consequente comprometimento da qualidade de vida dos ido$\operatorname{sos}^{1-4}$. Esse perfil exige, portanto, maior atuação dos serviços no sentido de minimizar esse impacto e garantir adequada qualidade de vida a esse crescente segmento da populaçãa ${ }^{5-7}$.

Embora o conceito de incapacidade inclua deficiência nas funções ou nas estruturas do corpo, limitações para execução de atividades e restrições de participação em diferentes situações de vida, além de considerar as circunstâncias nas quais o indivíduo vive ${ }^{8}$, na prática, essa condição é comumente avaliada pelas medidas de limitações nas atividades do cotidiano, envolvendo atividades básicas e instrumentais de vida diária (ABVD e AIVD, respectivamente) e mobilidade ${ }^{9-14}$.

Utilizando-se desse conceito, diversos estudos têm abordado a associação da incapacidade com algumas doenças crônicas, destacando-se as cardiovasculares, como infarto, angina, hipertensão arterial e acidente vascular encefálico (AVE), e o diabetes, que são frequentemente apontadas como associadas a diferentes níveis de incapacidade entre idosos, residentes tanto no Brasil ${ }^{15,16}$ como em outros países ${ }^{17-19}$. Além disso, é importante considerar o efeito da ocorrência simultânea de várias doenças na determinação da incapacidade de idosos, considerando as evidências observadas em outros países ${ }^{20-24}$, mas escassa no Brasil, dado que essa multimorbidade aumenta a gravidade da incapacidade e o risco de morte ${ }^{25-27}$. Na Suécia, estudo na população com 75 anos e mais mostrou que a prevalência de incapacidade nas atividades básicas apresentou importantes variações entre as diferentes combinações de doenças crônicas avaliadas ${ }^{23}$, enquanto na Austrália foi possível observar associação mais evidente das doenças cardiovasculares com a incapacidade nas atividades básicas em comparação às instrumentais $^{28}$. Portanto, a literatura ainda apresenta resultados divergentes, não sendo possível traçar, até o momento, um perfil de multimorbidade associado a maior ocorrência de incapacidade em seus diferentes domínios, sobretudo para o grupo de condições cardiovasculares ${ }^{23,27,28}$, que se apresenta como importante determinante da funcionalidade nesse grupo populacional $1^{19,20}$.

Especificamente na população brasileira, estudos anteriores já demonstraram a importante carga das doenças cardiovasculares e diabetes, entre outras, para a população, em termos de expectativa de vida e anos de vida vividos com incapacidade ou perdidos por morte prematura ${ }^{29-31}$, evidenciando o potencial das estratégias de controle desses agravos para a compressão da morbidade entre idosos ${ }^{32}$. No entanto, poucos estudos avaliaram o impacto da multimorbidade para a incapacidade ${ }^{33-35}$, utilizando apenas o número de doenças como variável de exposição, mas não detalhando as associações entre a incapacidade e cada combinação específica dessas condições crônicas. Além disso, a grande parte dos estudos brasileiros avaliam a incapacidade em apenas um domínio $^{35}$ ou a ocorrência de limitações em diferentes domínios ao mesmo tempo ${ }^{33,34}$, podendo não demonstrar os diferentes efeitos entre cada doença e suas combinações nos diferentes níveis que compõem a incapacidade para o desempenho de tarefas habituais.

Nesse sentido, o presente estudo teve por objetivo investigar as associações da incapacidade, avaliada em três níveis (ABVD, AIVD e mobilidade) com doenças cardiovasculares e diabetes, em uma amostra representativa de idosos residentes na Região Metropolitana de Belo Horizonte $(\mathrm{RMBH})$. Adicionalmente, verificou-se a influência do perfil de multimorbidade, considerando as doenças investigadas, na incapacidade dessa população idosa.

\section{Metodologia}

\section{População e amostra}

Trata-se de um estudo transversal, baseado nos dados coletados no Inquérito de Saúde da Região Metropolitana de Belo Horizonte (RMBH), constituída por 34 municípios e uma população de 4,8 milhões de habitantes, conduzido em 2010. Os participantes foram selecionados por meio de amostra probabilística de conglomerados, estratificada em dois estágios. Os setores censitários do Instituto Brasileiro de Geografia e Estatística (IBGE) foram utilizados como unidade primária de seleção, e a unidade amostral foi o domicílio. Todos os residentes dos domicílios amostrados com idade superior ou igual a 20 anos foram elegíveis para entrevista face a face, que se deu por meio de um questionário suplementar à Pesquisa de Emprego e Desemprego na Região Metropolitana de Belo Horizonte (PED/ RMBH), realizada pela Fundação João Pinheiro, órgão do Governo de Estado de Minas Gerais. O 
questionário incluiu: características demográficas e socioeconômicas; condições de saúde autorreferidas, incluindo questões relativas à funcionalidade; comportamentos em saúde; realização de exames preventivos ou de rastreamento e uso de serviços de saúde. Outras informações podem ser encontradas em publicação anterior ${ }^{36}$. Para a presente análise foram selecionados todos os 2.172 indivíduos com 60 anos ou mais de idade, que fizeram parte do referido inquérito.

O Inquérito de Saúde da Região Metropolitana de Belo Horizonte foi aprovado pelo Comitê de Ética em Pesquisas do Centro de Pesquisas René Rachou, da Fundação Oswaldo Cruz em Minas Gerais, e todos os participantes assinaram um termo de consentimento livre e esclarecido.

\section{Variáveis do estudo}

Em relação à incapacidade, as questões foram agrupadas em três blocos ou níveis: atividades básicas de vida diária - ABVD (tomar banho; vestir-se; ir ao banheiro; transferir-se da cama para a cadeira e alimentar-se $)^{37}$; atividades instrumentais de vida diária - AIVD (fazer compras; preparar sua alimentação; realizar tarefas domésticas; utilizar o transporte público; tomar seus medicamentos; lidar com suas finanças) ${ }^{38}$; e mobilidade (caminhar dois quarteirões, subir dez degraus; inclinar-se/agachar-se; caminhar de um cômodo a outro) ${ }^{39}$. Cada pergunta foi avaliada como o grau de dificuldade para realizar a atividade, considerando as categorias: nenhuma dificuldade; alguma dificuldade; muita dificuldade; e realiza a atividade só com a ajuda de outra pessoa ou aparelho. Para este estudo, os idosos que relataram muita dificuldade ou que realizavam a atividade apenas com ajuda de outra pessoa ou aparelho foram considerados incapazes, para cada nível (ABVD, AIVD e mobilidade).

Em relação às condições cardiovasculares, foram incluídos os relatos de hipertensão, AVE, infarto agudo do miocárdio e angina pectoris, além do diabetes, considerado um importante fator de risco cardiovascular, baseando-se na pergunta "algum médico ou profissional de saúde disse que o senhor tinha...."

Como potenciais fatores de confusão, foram consideradas variáveis sociodemográficas (idade, sexo, escolaridade), comportamentos em saúde (tabagismo atual e consumo abusivo de álcool), condições musculoesqueléticas (artrite e doença na coluna), uso de serviços de saúde (número de consultas médicas e hospitalizações nos doze meses precedentes à entrevista) e cobertura por plano privado de saúde. A idade (60 a 69, 70 a 79 e 80 anos ou mais) e a escolaridade $(<4,4$ a 7 e 8 ou mais anos de estudo) foram categorizadas. Foram considerados como fumantes atuais os idosos que relataram consumir cigarros no momento da entrevista, independente da frequência e quantidade, e o consumo abusivo de bebida alcoólica foi considerado como o consumo de 5 ou mais doses em um único dia, nos trinta dias anteriores à entrevista. A seleção de variáveis como potenciais fatores de confusão foi baseada em estudos prévios, que demonstraram sua associação com incapacidade e condições crônicas ${ }^{2,4,11,16}$, ressaltando a importância de se considerar a maior probabilidade de uso de serviços de saúde, tanto entre idosos com incapacidade quanto entre aqueles com maior número de condições crônicas $^{2,5,11,26 .}$.

\section{Análise dos dados}

Primeiramente, foi realizada uma análise descritiva do perfil dos idosos participantes, considerando as características sociodemográficas, comportamentos em saúde, uso de serviços de saúde e existência de plano privado de saúde, bem como a prevalência da incapacidade em cada nível e das doenças investigadas. Posteriormente, avaliou-se a associação entre a incapacidade em cada domínio e todas as demais variáveis incluídas no estudo, utilizando-se o teste do qui-quadrado de Pearson e um nível de significância de $5 \%$.

A existência de associação entre o relato de incapacidade funcional e cada condição de saúde selecionada, bem como as combinações entre essas condições (para as combinações com prevalências superiores a $1 \%$ ), foi avaliada pelo cálculo das razões de prevalência (RP) e respectivos intervalos de confiança (95\%), utilizando o modelo de regressão de Poisson com variância robusta, ajustado pelas demais variáveis incluídas no estudo e descritas nas Tabelas 1 e 2, para cada domínio de incapacidade avaliado (ABVDs, AIVDs e mobilidade). Nos modelos de regressão que consideraram as combinações das morbidades como exposições, o grupo de idosos que reportaram todas as doenças descritas em cada combinação foi considerado exposto e os demais, independente das condições de saúde apresentadas, foram considerados pertencentes ao grupo não exposto, para estimativa das RP, sendo as demais variáveis incluídas como fatores de confusão.

Posteriormente, foi estimada a fração atribuível populacional (FAP) e os respectivos interva- 
Tabela 1. Características da amostra e prevalência de incapacidade segundo fatores sociodemográficos, comportamentais e uso de serviços de saúde, entre idosos residentes na Região Metropolitana de Belo Horizonte, 2010.

\begin{tabular}{|c|c|c|c|}
\hline \multirow{2}{*}{ Características (\% na amostra) } & \multicolumn{3}{|c|}{ Prevalência de incapacidade - \% } \\
\hline & ABVD & AIVD & Mobilidade \\
\hline \multicolumn{4}{|l|}{ Sexo } \\
\hline Feminino $(61,0)$ & $10,3^{\mathrm{a}}$ & $23,5^{\mathrm{a}}$ & $24,7^{\mathrm{a}}$ \\
\hline Masculino $(39,0)$ & 4,2 & 14,0 & 12,6 \\
\hline \multicolumn{4}{|l|}{ Faixa etária em anos } \\
\hline $60-69(55,4)$ & $3,0^{\mathrm{a}}$ & $9,7^{\mathrm{a}}$ & $11,1^{\mathrm{a}}$ \\
\hline $70-79(30,2)$ & 7,9 & 23,1 & 23,2 \\
\hline 80 ou mais $(14,4)$ & 26,6 & 51,4 & 47,0 \\
\hline \multicolumn{4}{|l|}{ Escolaridade em anos } \\
\hline$<4(26,0)$ & $11,7^{\mathrm{a}}$ & $31,4^{\mathrm{a}}$ & $30,0^{\mathrm{a}}$ \\
\hline $4-7(37,7)$ & 8,0 & 19,7 & 20,8 \\
\hline 8 ou mais $(36,3)$ & 5,1 & 11,6 & 11,9 \\
\hline \multicolumn{4}{|l|}{ Tabagismo atual } \\
\hline Não $(89,9)$ & $8,5^{\mathrm{a}}$ & $20,5^{\mathrm{a}}$ & 20,2 \\
\hline $\operatorname{Sim}(10,1)$ & 2,8 & 13,3 & 17,7 \\
\hline \multicolumn{4}{|l|}{ Consumo de bebida alcoólica } \\
\hline Não $(80,9)$ & $9,6^{\mathrm{a}}$ & $22,3^{\mathrm{a}}$ & $22,6^{\mathrm{a}}$ \\
\hline $\operatorname{Sim}(19,1)$ & 1,4 & 10,5 & 10,2 \\
\hline \multicolumn{4}{|l|}{ Plano de saúde } \\
\hline Não $(53,7)$ & 8,3 & $21,4^{\mathrm{a}}$ & $21,9^{a}$ \\
\hline $\operatorname{Sim}(46,3)$ & 7,0 & 17,5 & 17,4 \\
\hline \multicolumn{4}{|l|}{ Hospitalização no último ano } \\
\hline Não $(90,9)$ & $6,7^{\mathrm{a}}$ & $17,4^{\mathrm{a}}$ & $18,1^{\mathrm{a}}$ \\
\hline $\operatorname{Sim}(9,1)$ & 20,0 & 43,8 & 37,7 \\
\hline \multicolumn{4}{|l|}{ Consultas médicas no último ano } \\
\hline Nenhuma $(21,1)$ & $3,9^{\mathrm{a}}$ & $13,0^{\mathrm{a}}$ & $14,3^{\mathrm{a}}$ \\
\hline 1 ou $2(32,2)$ & 5,3 & 14,9 & 13,5 \\
\hline 3 ou mais $(46,7)$ & 11,4 & 26,2 & 26,8 \\
\hline
\end{tabular}

los de confiança, também para cada condição (e combinações) em cada domínio da incapacidade, utilizando-se o comando "punaf", do Stata, que utiliza o método de Greenland e Drescher ${ }^{40}$, considerando o modelo ajustado por todos os fatores de confusão. Esse método compara o cenário observado no estudo com aquele que seria observado, caso a variável de exposição assumisse o valor zero (ausência das doenças). Portanto, os valores estimados nessa análise indicam a proporção da prevalência da incapacidade que poderia ser evitada pela eliminação das doenças ou suas combinações na população estudada, mantendo-se as demais características consideradas no modelo.

Todas as análises foram realizadas no programa Stata 13.0, utilizando-se os procedimentos para análise de inquéritos com amostras complexas.

\section{Resultados}

Entre os idosos participantes do inquérito, 7,9\% (IC95\%: 6,8\% - 9,2\%) apresentavam incapacidade nas ABVD's, 19,8\% (IC95\%: 18,0\% - 21,8\%) eram incapazes nas AIVD's e 19,9\% (IC95\%: $18,1 \%$ - 21,9\%) eram dependentes no domínio mobilidade.

A Tabela 1 mostra a descrição dos participantes da amostra quanto às características sociodemográficas, comportamentais, uso de serviços de saúde e existência de plano privado de saúde, e a associação dessas variáveis com a incapacidade. Em resumo, observou-se predomínio de mulheres, de idosos com 60 a 69 anos, com mais de 4 anos de estudo, que não fumavam e não consumiam bebidas alcoólicas de forma excessiva, que 
não foram hospitalizados, mas que realizaram pelo menos uma consulta médica no último ano. Foi possível observar ainda que todas as variáveis apresentaram associação significativa $(\mathrm{p}<0,05)$ com os três níveis de incapacidade pesquisados, com exceção para plano privado de saúde com ABVD, e tabagismo atual com mobilidade.

A Tabela 2 mostra a prevalência das condições de saúde e a associação com cada nível de incapacidade avaliado. A doença mais frequente foi hipertensão arterial, seguida por doença da coluna, artrite e diabetes. A prevalência de incapacidade, para todos os níveis, foi significativamente maior entre os indivíduos que relataram as doenças avaliadas, com exceção para a prevalência de ABVD entre diabéticos e não diabéticos.

A descrição dos participantes da amostra quanto ao perfil de multimorbidade e a associação com incapacidade pode ser vista na Tabela 3. Entre as combinações de doenças pesquisadas, a maior prevalência foi para ocorrência simultânea de hipertensão arterial e diabetes (12,2\%), seguida pela ocorrência de hipertensão e infarto/ Angina (4,9\%) e de hipertensão e $\operatorname{AVE}(3,3 \%)$. A prevalência de incapacidade, em todos os níveis, foi significativamente maior entre os idosos que apresentaram as multimorbidades descritas na tabela.
Os resultados das associações (bruta e ajustada) entre as condições de saúde (e suas combinações) e cada nível de incapacidade considerado nesse estudo estão apresentados na Tabela $4 . \mathrm{Na}$ análise não ajustada todas as associações foram estatisticamente significativas, indicando maior prevalência de incapacidade entre os idosos que relataram uma ou mais doenças crônicas. Após o ajustamento e considerando cada doença isoladamente, o infarto/angina apresentou associação significativa com incapacidade em AIVD (RP: 1,46; IC95\%: 1,11-1,92) e o AVE apresentou associação consistente com os três níveis de incapacidade: ABVD (RP: 3,34; IC95\%: 2,08-5,38), AIVD (RP: 1,62; IC95\%: 1,14-2,30) e mobilidade (RP: 1,81; IC95\%: 1,28-2,54). Quando o perfil de multimorbidade foi analisado, a combinação entre hipertensão e AVE foi associada com a incapacidade nas ABVD's (RP: 2,65; IC95\%: 1,54-4,56); os idosos com hipertensão e infarto/angina apresentaram maior prevalência de incapacidade em AIVD's (RP: 1,52; IC95\%: 1,12-2,07) e em mobilidade (RP: 1,38; IC95\%: 1,00-1,92); a simultaneidade de diabetes e infarto/angina aumentou a prevalência de incapacidade nos três níveis (RP: 1,92; IC95\%: 1,14-3,23 para ABVD, RP: 2,01; IC95\%: 1,39-2,91 para AIVD e RP: 1,80; IC95\%: 1,26-2,57 para mobilidade); e o relato de três

Tabela 2. Prevalência da incapacidade segundo as doenças crônicas selecionadas, entre idosos residentes na Região Metropolitana de Belo Horizonte, 2010.

\begin{tabular}{|c|c|c|c|}
\hline \multirow{2}{*}{ Doenças crônicas (\% na amostra) } & \multicolumn{3}{|c|}{ Prevalência de incapacidade - \% } \\
\hline & ABVD & AIVD & Mobilidade \\
\hline \multicolumn{4}{|l|}{ Hipertensão arterial } \\
\hline Não $(43,9)$ & $6,4^{\mathrm{a}}$ & $15,1^{\mathrm{a}}$ & $15,4^{\mathrm{a}}$ \\
\hline $\operatorname{Sim}(56,1)$ & 9,2 & 23,6 & 23,5 \\
\hline \multicolumn{4}{|l|}{ Diabetes } \\
\hline Não $(84,2)$ & 7,4 & $18,2^{\mathrm{a}}$ & $18,6^{\mathrm{a}}$ \\
\hline $\operatorname{Sim}(15,8)$ & 10,9 & 28,6 & 27,0 \\
\hline \multicolumn{4}{|l|}{ Infarto/angina } \\
\hline Não $(96,0)$ & $7,1^{\mathrm{a}}$ & $18,1^{\mathrm{a}}$ & $18,6^{\mathrm{a}}$ \\
\hline $\operatorname{Sim}(4,0)$ & 20,7 & 45,6 & 39,4 \\
\hline \multicolumn{4}{|l|}{ Acidente vascular encefálico } \\
\hline Não $(95,3)$ & $6,6^{\mathrm{a}}$ & $18,1^{\mathrm{a}}$ & $18,4^{\mathrm{a}}$ \\
\hline $\operatorname{Sim}(4,7)$ & 36,5 & 54,7 & 50,8 \\
\hline \multicolumn{4}{|l|}{ Artrite } \\
\hline Não $(83,0)$ & $6,8^{\mathrm{a}}$ & $17,1^{\mathrm{a}}$ & $17,6^{\mathrm{a}}$ \\
\hline $\operatorname{Sim}(17,0)$ & 13,7 & 33,1 & 31,7 \\
\hline \multicolumn{4}{|l|}{ Doença da coluna } \\
\hline Não $(82,4)$ & $7,3^{\mathrm{a}}$ & $18,4^{\mathrm{a}}$ & $17,5^{\mathrm{a}}$ \\
\hline $\operatorname{Sim}(17,6)$ & 11,1 & 26,6 & 31,6 \\
\hline
\end{tabular}

ABVD: atividades básicas da vida diária; AIVD: atividades instrumentais da vida diária.

${ }^{\mathrm{a}} \mathrm{p}<0,05$ para teste do qui-quadrado de Pearson. 
Tabela 3. Prevalência de incapacidade segundo o perfil de multimorbidade, entre idosos residentes na Região Metropolitana de Belo Horizonte, 2010.

\begin{tabular}{|c|c|c|c|}
\hline \multirow{2}{*}{ Multimorbidade (\% na amostra) } & \multicolumn{3}{|c|}{ Prevalência de incapacidade - \% } \\
\hline & ABVD & AIVD & Mobilidade \\
\hline \multicolumn{4}{|l|}{ Hipertensão e diabetes } \\
\hline Não $(87,8)$ & $7,3^{\mathrm{a}}$ & $18,4^{\mathrm{a}}$ & $18,7^{\mathrm{a}}$ \\
\hline $\operatorname{Sim}(12,2)$ & 12,1 & 30,4 & 28,7 \\
\hline \multicolumn{4}{|l|}{ Hipertensão e infarto/angina } \\
\hline Não $(95,1)$ & $7,2 \mathrm{a}$ & $18,3^{\mathrm{a}}$ & $18,7^{\mathrm{a}}$ \\
\hline $\operatorname{Sim}(4,9)$ & 21,1 & 48,4 & 42,8 \\
\hline \multicolumn{4}{|l|}{ Hipertensão e AVE } \\
\hline Não $(96,7)$ & $6,9^{\mathrm{a}}$ & $18,7^{\mathrm{a}}$ & $19,0^{\mathrm{a}}$ \\
\hline $\operatorname{Sim}(3,3)$ & 38,3 & 53,8 & 48,3 \\
\hline \multicolumn{4}{|l|}{ Diabetes e infarto/angina } \\
\hline Não $(98,4)$ & $7,6^{\mathrm{a}}$ & $19,1^{\mathrm{a}}$ & $19,3^{\mathrm{a}}$ \\
\hline $\operatorname{Sim}(1,6)$ & 27,6 & 61,8 & 54,6 \\
\hline \multicolumn{4}{|l|}{ Diabetes e AVE } \\
\hline Não $(98,7)$ & $7,7^{\mathrm{a}}$ & $19,4^{\mathrm{a}}$ & $19,6^{\mathrm{a}}$ \\
\hline $\operatorname{Sim}(1,3)$ & 28,7 & 48,5 & 46,8 \\
\hline \multicolumn{4}{|l|}{ Hipertensão, diabetes e infarto/angina } \\
\hline Não $(98,6)$ & $7,7^{\mathrm{a}}$ & $19,2^{\mathrm{a}}$ & $19,5^{\mathrm{a}}$ \\
\hline $\operatorname{Sim}(1,4)$ & 20,9 & 60,7 & 52,4 \\
\hline \multicolumn{4}{|l|}{ Hipertensão, diabetes e AVE } \\
\hline Não $(98,9)$ & $7,7^{\mathrm{a}}$ & $19,4^{\mathrm{a}}$ & $19,6^{\mathrm{a}}$ \\
\hline $\operatorname{Sim}(1,1)$ & 31,4 & 54,2 & 52,2 \\
\hline
\end{tabular}

doenças (hipertensão, diabetes e infarto/angina) foi associado a maior prevalência de incapacidade em AIVD (RP: 2,25; IC95\%: 1,51-3,34) e em atividades de mobilidade (RP: 1,96; IC95\%: 1,33-2,90).

A Tabela 5 descreve a fração atribuível populacional (e intervalos com 95\% de confiança), que demonstra a proporção da prevalência da incapacidade em cada domínio que pode ser explicada pela ocorrência da doença ou da combinação de doenças. De forma semelhante ao observado na tabela anterior, o infarto/angina apresentou contribuição significativa $(4,6 \%)$ para a prevalência de AIVD, enquanto o AVE contribuiu significativamente para a prevalência da incapacidade em todos os domínios $(15,0 \%$ para ABVD, $4,8 \%$ para AIVD e 5,3\% para mobilidade). A ocorrência simultânea de hipertensão e AVE apresenta contribuição significativa para a prevalência de incapacidade em ABVD (10,0\%), enquanto as doenças coronarianas (infarto/angina) foram importantes apenas para as prevalências de incapacidade em AIVD e em mobilidade, quando combinados com hipertensão, diabetes e com hipertensão e diabetes, de forma simultânea.

\section{Discussão}

Os resultados do presente estudo, conduzido em uma amostra representativa de idosos residentes em uma grande região metropolitana brasileira, contribuem para o conhecimento da carga de incapacidade e da associação desse desfecho com eventos cardiovasculares e diabetes. Esses resultados indicam que: (i) a população estudada apresentou uma expressiva prevalência de incapacidade, sobretudo nas atividades instrumentais (AIVD) e nas atividades de mobilidade, que atingiram quase um quinto dos idosos; (ii) avaliadas individualmente, apenas o relato de infarto e/ou angina e de AVE mostraram-se associados à incapacidade, sendo que a presença dessa última condição apresentou contribuição significativa para a prevalência da incapacidade nos três domínios avaliados; (iii) o perfil de multimorbi- 


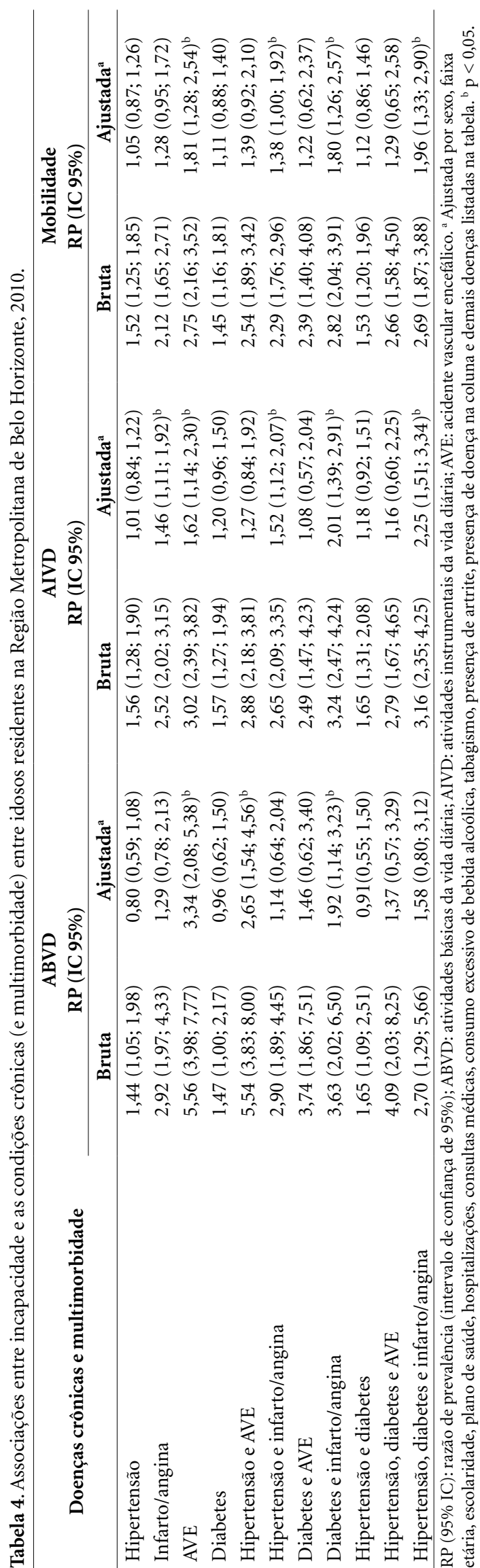

dade mostrou-se importante para a ocorrência de incapacidade nessa população, com destaque para hipertensão, diabetes e infarto/angina.

Embora a comparação dos valores de prevalência com outros inquéritos é dificultada pelas diferenças na definição do evento ${ }^{17}$, a prevalência de incapacidade em ABVD se aproxima daquelas relatadas entre idosos brasileiros, nos inquéritos nacionais de 1998, 2003 e 2008, considerando três atividades (alimentar-se, tomar banho e usar o banheiro), que foram de $6,5 \%, 6,4 \%$ e $6,9 \%$ respectivamente $^{41}$. Já a prevalência de incapacidade para mobilidade entre idosos brasileiros foi inferior ao observado no presente estudo $(7,8 \%$ em 1998 e 7,6\% em 2003) ${ }^{42}$, mas utilizando-se apenas uma variável para essa definição ("dificuldade para caminhar cerca de $100 \mathrm{~m}$ "), o que pode justificar essa expressiva diferença. De qualquer forma, é evidente que a prevalência de incapacidade relacionada às AIVDs e à mobilidade na população estudada é, possivelmente, maior quando comparada aos dados nacionais existentes, podendo gerar importantes impactos nas condições de vida dessa população.

Os resultados deste estudo demonstram que as condições cardiovasculares apresentam associação com incapacidade entre idosos, de forma semelhante ao observado em outras populações ${ }^{15,17,19,43}$, mas acrescenta por demonstrar a importância da multimorbidade para essa associação. Considerando as doenças analisadas neste estudo de forma isolada, o AVE e os eventos coronarianos (infarto/angina) foram as que apresentaram associação independente com incapacidade, mesmo considerando o ajustamento pelas demais variáveis, incluindo as demais condições de saúde. A associação da incapacidade com AVE, já descrita em alguns estudos no Brasil ${ }^{11,16,44,45} \mathrm{e}$ no mundo ${ }^{43,46}$, pode estar relacionada a complicações decorrentes da doença como a perda cognitiva, a depressão, as alterações na marcha e a incontinência urinária, complicações essas que podem afetar desde as atividades mais básicas, como se alimentar, até a participação social do idoso $^{16,47}$, o que explica a associação reportada neste estudo. Com relação ao infarto, a literatura também descreve essa associação com incapacidade $^{33,48}$, mas ressalta que a perda da funcionalidade na população acometida pelo infarto pode depender das características demográficas, da presença de depressão ou ansiedade, do encaminhamento ou não para a reabilitação e da própria cobertura por plano privado de saúde ${ }^{48,49}$, o que pode justificar resultados diversos entre populações. O que poderia explicar a relação de infarto/ 
Tabela 5. Fração atribuível populacional das condições crônicas (e multimorbidade) na prevalência da incapacidade nos três domínios entre idosos residentes na Região Metropolitana de Belo Horizonte, 2010.

\begin{tabular}{lrrr}
\hline \multirow{2}{*}{ Doenças crônicas e multimorbidade } & \multicolumn{3}{c}{ Fração atribuível populacional (IC 95\%) } \\
\cline { 2 - 4 } & \multicolumn{1}{c}{ ABVD } & AIVD & \multicolumn{1}{c}{ Mobilidade } \\
\hline Hipertensão & $-16,3(-41,3 ; 4,3)$ & $0,9(-11,8 ; 12,1)$ & $2,9(-9,6 ; 14,1)$ \\
Infarto/angina & $3,8(-4,4 ; 11,2)$ & $4,6(0,7 ; 8,3)^{\mathrm{b}}$ & $2,7(-1,0 ; 6,2)$ \\
AVE & $15,0(6,5 ; 22,7)^{\mathrm{b}}$ & $4,8(0,6 ; 8,8)^{\mathrm{b}}$ & $5,3(1,4 ; 9,0)^{\mathrm{b}}$ \\
Diabetes & $-0,8(-0,1 ; 7,7)$ & $3,7(-1,2 ; 8,4)$ & $2,1(-2,7 ; 6,7)$ \\
Hipertensão e AVE & $10,0(2,3 ; 17,2)^{\mathrm{b}}$ & $1,8(-1,7 ; 5,2)$ & $2,3(-1,0 ; 5,4)$ \\
Hipertensão e infarto/angina & $1,7(-6,1 ; 8,9)$ & $4,3(0,6 ; 7,8)^{\mathrm{b}}$ & $3,0(-0,4 ; 6,4)$ \\
Diabetes e AVE & $1,4(-2,2 ; 4,8)$ & $0,2(-1,7 ; 2,2)$ & $0,5(-1,4 ; 2,4)$ \\
Diabetes e infarto/angina & $2,9(-0,1 ; 5,8)$ & $2,7(0,8 ; 4,6)^{\mathrm{b}}$ & $2,1(0,5 ; 3,7)^{\mathrm{b}}$ \\
Hipertensão e diabetes & $-1,7(-10,7 ; 6,5)$ & $2,7(-1,6 ; 6,9)$ & $1,8(-2,6 ; 6,1)$ \\
Hipertensão, diabetes e AVE & $1,1(-2,4 ; 4,5)$ & $0,4(-1,6 ; 2,4)$ & $0,6(-1,3 ; 2,6)$ \\
Hipertensão, diabetes e infarto/angina & $1,5(-1,2 ; 4,0)$ & $2,6(0,8 ; 4,3)^{\mathrm{b}}$ & $1,9(0,5 ; 3,4)^{\mathrm{b}}$ \\
\hline IC95\%: intervalo de confiança de 95\%; ABVD: atividades básicas da vida diária; AIVD: atividades instrumentais da vida diária; \\
AVE: acidente vascular encefálico. ${ }^{\mathrm{a}}$ Fração atribuível populacional, considerando o modelo ajustado por sexo, faixa etária, \\
escolaridade, plano de saúde, hospitalizações, consultas médicas, consumo excessivo de bebida alcoólica, tabagismo, presença de \\
artrite, presença de doença na coluna e demais doenças listadas na tabela. ${ }^{\mathrm{b}} \mathrm{p}<0,05$.
\end{tabular}

angina apenas com as AIVDs no presente estudo, seria a ocorrência de quadros de menor gravidade dessas condições, considerando que já foi demonstrado que maiores complicações desses eventos estão relacionadas mais fortemente com a incapacidade ${ }^{50}$.

Por outro lado, os relatos de diabetes e hipertensão, quando analisados isoladamente, não estiveram associados a nenhum domínio da incapacidade. A associação do diabetes com incapacidade observada em outros estudos pode ser consequência de complicações da doença $a^{15,33,43,45,51}$ ou comorbidades ${ }^{52,53}$, como doenças cardíacas ${ }^{51}$. Portanto, o ajustamento pelas condições cardiovasculares poderia ter atenuado a magnitude das associações avaliadas ${ }^{15,52}$, hipótese que é reforçada pela observação de associação significativa para a combinação diabetes e infarto/angina, em todos os domínios, e para hipertensão, diabetes e infarto/angina para a incapacidade em AIVD e mobilidade. Sobre a não associação com o relato de hipertensão arterial, quando avaliado de forma isolada, Verbrugge et al. já haviam evidenciado que condições crônicas de saúde com alta prevalência geralmente possuem um baixo impacto sobre a incapacidade ${ }^{24}$ o que pode ter ocorrido entre os idosos da RMBH. Além disso, um estudo realizado em amostra representativa da população idosa brasileira evidenciou a redução da força de associação entre déficit na mobilidade e hipertensão arterial ao longo de 10 anos, o que pode ser explicado pela existência de políticas públicas voltadas para a prevenção das complicações da doença, reduzindo então a força de associação com o desfecho incapacidade ${ }^{54}$, o que poderia, também, justificar a não associação observada neste estudo.

Conforme mencionado anteriormente, a maioria dos estudos reportam associações entre cada doença, de forma isolada, e a incapacida$\mathrm{de}^{15,19,43,48,51}$ ou exploram o efeito do número de condições crônicas na prevalência e incidência desse desfecho entre idosos ${ }^{21-23}$. No entanto, é importante considerar o efeito de cada combinação de doenças na incapacidade, considerando que esse efeito pode variar segundo essas combinações, como já reportado em algumas populações ${ }^{20,23,27,55}$.

As evidências produzidas até o momento mostram, no entanto, ampla variação de resultados sobre a associação do perfil de multimorbidade com incapacidade entre idosos, sendo que um perfil de maior vulnerabilidade ainda não foi consistentemente definido na literatura, o que justifica a identificação dessas associações em diferentes populações. Ademais, apesar do reconhecido impacto das doenças cardiovasculares e de seus fatores de risco, como o diabetes, para a incapacidade entre idosos ${ }^{17,19}$, poucos estudos exploraram, especificamente, o efeito das combinações desses agravos. Estudo conduzido entre idosos residentes na Suécia evidenciou associação significativa entre a incapacidade em atividades básicas e a ocorrência simultânea de AVE e hipertensão, mas não foi observada associação com a ocorrência de doença coronariana e 
hipertensão, doença coronariana e diabetes, além de hipertensão e diabetes ${ }^{23}$. Por outro lado, entre mulheres idosas norte-americanas, as ocorrências combinadas de AVE e doenças coronarianas, AVE e hipertensão, e doenças coronarianas e diabetes foram perfis significativamente associados à prevalência de incapacidade ${ }^{27}$. Reforçando esses achados, estudo longitudinal conduzido na população idosa da Austrália identificou três perfis de multimorbidade importantes na determinação da incapacidade em atividades básicas de vida diária, sendo um desses perfis constituído por eventos coronarianos e $\mathrm{AVE}^{28}$. No Brasil, estudos anteriores demonstraram a importância do AVE na determinação de limitações em atividades básicas ${ }^{56}$ e instrumentais ${ }^{35}$ entre idosos acompanhados por seis anos. No entanto, apenas uma publicação explorou o impacto da combinação do diabetes e AVE na ocorrência de limitações nas atividades básicas, mas não investigou outros domínios da incapacidade ou o impacto de outras combinações de doenças ${ }^{55}$. Nossos resultados acrescentam por demonstrar a importância dessas combinações na associação com incapacidade em uma amostra de idosos residentes no Brasil, corroborando com os estudos que demonstraram o papel das doenças coronarianas no perfil de multimorbidade ${ }^{23}$, além do diabetes e hipertensão, quando combinados às demais condições. Além disso, a quantificação da proporção do peso da incapacidade que pode ser atribuída a cada doença e a cada combinação estudada, chama atenção para a importância do direcionamento de medidas de prevenção e controle para esses agravos. Ademais, os resultados descritos aqui demonstram variações dessas combinações em relação ao domínio da incapacidade, se associando de forma diferente às limitações em ABVD, AIVD e mobilidade.

O presente estudo é de natureza transversal, o que não permite estabelecer relações temporais entre as variáveis estudadas. Além disso, as condições de saúde e a dificuldade na execução das tarefas investigadas foram avaliadas por questões autorreferidas, o que pode levar a um viés de informação, embora estudos prévios tenham demonstrado adequada validade dessas ques- tões ${ }^{57,58}$. Além disso, o delineamento deste estudo favorece o viés de sobrevivência, o que poderia, de certa forma, subestimar as associações analisadas, sobretudo para aquelas condições de elevada mortalidade, como o AVE, e, consequentemente, reduzir as estimativas da fração atribuível populacional apresentadas. Por outro lado, pelo nosso conhecimento, esse é o primeiro estudo brasileiro a descrever as associações da incapacidade com combinações de doenças cardiovasculares e diabetes, condições comuns entre idosos e com expressivo impacto na incapacidade, permitindo a identificação de grupos mais vulneráveis às limitações das atividades em três domínios diferentes (ABVD, AIVD e mobilidade). Além disso, trata-se de um estudo conduzido em uma amostra representativa de idosos residentes em uma grande região metropolitana brasileira, que considerou os possíveis fatores de confusão para a verificação das associações pesquisadas.

Portanto, de maneira geral, os resultados das combinações das condições cardiovasculares estudadas mostram a necessidade de maior atenção aos idosos acometidos por múltiplas condições de saúde, perfil comum nesse grupo populacional $^{2,7,10}$, chamando atenção para a maior contribuição do AVE, de forma isolada ou combinada com a hipertensão, para as atividades básicas e da maior importância das doenças coronarianas (infarto e/ou angina), sobretudo quando ocorre de forma simultânea com diabetes ou com diabetes e hipertensão, para a prevalência de incapacidade nas atividades instrumentais e na mobilidade. Esses achados demonstram a relevância do acompanhamento contínuo de idosos com um perfil de multimorbidade de maior vulnerabilidade, conforme descrito anteriormente, o que deve incluir avaliações de diferentes profissionais e a possível inclusão em programas de reabilitação, como já demonstrado anteriormente ${ }^{59}$. Vale destacar também a importância dos programas de prevenção dos fatores de risco para doenças como o AVE e o infarto, considerando o elevado impacto sobre a capacidade funcional, o que poderia evitar a ocorrência desses agravos e, consequentemente, a maior necessidade de cuidado, incluindo a institucionalização ${ }^{23,60}$. 


\section{Colaboradores}

GM Bernardes participou da concepção do estudo, análise dos dados, interpretação dos resultados e redação do trabalho; JVM Mambrini participou da análise dos dados, interpretação dos resultados e revisão final do artigo; MF Lima-Costa participou da concepção e delineamento do estudo, interpretação dos resultados e revisão final do artigo; SV Peixoto participou da concepção do estudo, análise dos dados, interpretação dos resultados e revisão final do artigo.

\section{Agradecimentos}

GMB foi bolsista de mestrado da Coordenação de Aperfeiçoamento de Pessoal de Nível Superior (CAPES). MFL-C e SVP são bolsistas de produtividade do Conselho Nacional de Desenvolvimento Científico e Tecnológico (CNPq).

\section{Referências}

1. Alves LC, Leite IC, Machado CJ. Conceituando e mensurando a incapacidade funcional da população idosa: uma revisão de literatura. Cien Saude Colet 2008; 13(4):1199-1207.

2. Fialho CB, Lima-Costa MF, Giacomin KC, Loyola Filho AI. Capacidade funcional e uso dos serviços de saúde por idosos da Região Metropolitana de Belo Horizonte, Minas Gerais, Brasil: um estudo de base populacional. Cad Saude Publica 2014; 30(3):599-610.

3. Fiedler MM, Peres KG. Capacidade funcional e fatores associados em idosos do Sul do Brasil: um estudo de base populacional. Cad Saude Publica 2008; 24(2):409415.

4. Alves LC, Leite IC, Machado CJ. Fatores associados à incapacidade funcional dos idosos no Brasil: análise multinível. Rev Saude Publica 2010; 44(3):468-478.

5. Lima-Costa MF, Facchini LA, Matos DL, Macinko J. Mudanças em dez anos das desigualdades sociais em saúde dos idosos brasileiros (1998-2008). Rev Saude Publica 2012; 46(Supl. 1):100-107.

6. Rebouças M, Matos MR, Ramos LR. O que há de novo em ser velho. Saude Soc 2013; 22(4):1226-1235.

7. Veras R. Envelhecimento populacional contemporâneo: demandas, desafios e inovações. Rev Saude Publica 2009; 43(3):548-554.

8. Organização Mundial de Saúde (OMS). Classificação Internacional de Funcionalidade, Incapacidade e Saúde. São Paulo: EDUSP; 2003.

9. Nagi SZ. An epidemiology of disability among adults in the United States. Milbank Mem Fund Q Health Soc 1976; 54(4):439-467.

10. Verbrugge LM, Jette AM. The disablement process. Soc Sci Med 1994; 38(1):1-14.

11. Giacomin KC, Peixoto SV, Uchoa E, Lima-Costa MF Estudo de base populacional dos fatores associados à incapacidade funcional entre idosos na Região Metropolitana de Belo Horizonte, Minas Gerais, Brasil. Cad Saude Publica 2008; 24(6):1260-1270.

12. Neri AL, Yassuda MS, Araújo LF, Eulálio MC, Cabral BE, Siqueira MEC, Santos GA, Moura JGA. Metodologia e perfil sociodemográfico cognitivo e de fragilidade em idosos comunitários de sete cidades brasileiras: Estudo FIBRA. Cad Saude Publica 2013; 29(4):778-792.

13. Nogueira SL, Ribeiro RCL, Rosado LEFPL, Franceschini SCC, Ribeiro AQ, Pereira ET. Fatores determinantes da capacidade funcional em idosos longevos. Rev Bras Fisioter 2010; 14(4):322-329.

14. Parahyba MI, Veras R. Diferenciais sociodemográficos no declínio funcional em mobilidade física entre os idosos no Brasil. Cien Saude Colet 2008; 13(4):12571264.

15. Alves LC, Leimann BCQ, Vasconcelos MEL, Carvalho MS, Vasconcelos AGG, Fonseca TCO, Lebrão ML, Laurenti R. A influência das doenças crônicas na capacidade funcional dos idosos do Município de São Paulo, Brasil. Cad Saude Publica 2007; 23(8):1924-1930.

16. Barbosa BR, Almeida JM, Barbosa MR, Rossi-Barbosa LAR. Avaliação da capacidade funcional dos idosos e fatores associados à incapacidade. Cien Saude Colet 2014; 19(8):3317-3325.

17. Kattainen A, Koskinen S, Reunanen A, Martelin T, Knekt P, Aromaa A. Impact of cardiovascular diseases on activity limitations and need for help among older persons. J Clin Epidemiol 2004; 57(1):82-88. 
18. Quinones PA, Seidl H, Holle R, Kuch B, Meisinger C, Hunger M, Kirchberger I. New potential determinants of disability in aged persons with myocardial infarction: results from the KORINNA-study. BMC Geriatr 2014; 14:34.

19. Welmer A, Angleman S, Rydwik E, Fratiglioni L, Qiu C. Association of cardiovascular burden with mobility limitation among elderly people: a population-based study. PLoS One 2013; 8(5):e65815.

20. Griffith L, Raina P, Wu H, Zhu B, Stathokostas L. Population attributable risk for functional disability associated with chronic conditions in Canadian older adults. Age Ageing 2010; 39(6):738-745.

21. Guralnik JM, Lacroix AZ, Everett DF, Kovar MG. Aging in the eighties: the prevalence of comorbidity and its association with disability. Washington: Department of Health and Human Services, Public Health Service, Centers for Disease Control, National Center for Health Statistics; 1989.

22. Marengoni A, Strauss E, Rizzuto D, Winblad B, Fratiglioni L. The impact of chronic multimorbidity and disability on functional decline and survival in elderly persons. A community-based, longitudinal study. J Intern Med 2008; 265(2):288-295.

23. Marengoni A, Angleman S, Fratiglioni L. Prevalence of disability according to multimorbidity and disease clustering: a population-based study. Journal of Comorbidity 2011; 1(1):11-18.

24. Verbrugge LM, Lepkowski JM, Imanaka Y. Comorbidity and its impact on disability. Milbank Q 1989; 67(34):450-484.

25. Landi F, Liperoti R, Russo A, Capoluongo E, Barillaro C, Pahor M, Bernabei R, Onder G. Disability, more than multimorbidity, was predictive of mortality among older persons aged 80 years and older. J Clin Epidemiol 2010; 63(7):752-759.

26. Salive ME. Multimorbidity in older adults. Epidemiol Rev 2013; 35:75-83.

27. Fried LP, Bandeen-Roche K, Kasper JD, Guralnik JM. Association of comorbidity with disability in older women: the women's health and aging study. J Clin Epidemiol 1999; 52(1):27-37.

28. Jackson CA, Jones M, Tooth L, Mishra GD, Byles J, Dobson A. Multimorbidity patterns are differentially associated with functional ability and decline in a longitudinal cohort of older women. Age Ageing 2015; 44(5):810-816.

29. Campolina AG, Adami F, Santos JLF, Lebrão ML. A transição de saúde e as mudanças na expectativa de vida saudável da população idosa: possíveis impactos da prevenção de doenças crônicas. Cad Saude Publica 2013; 29(6):1217-1229.

30. Leite IC, Valente JG, Schramm JM, Daumas RP, Rodrigues RN, Santos MF, Oliveira AF, Silva RS, Campos MR, Mota JC. Burden of disease in Brazil and its regions, 2008. Cad Saude Publica 2015; 31(7):1551-1564.

31. Costa AF, Flor LS, Campos MR, Oliveira AF, Costa MF, Silva RS, Lobato LC, Schramm JM. Burden of type 2 diabetes mellitus in Brazil. Cad Saude Publica 2017; 33(2):e00197915.

32. Campolina AG, Adami F, Santos JL, Lebrão ML. Effect of the elimination of chronic diseases on disability-free life expectancy among elderly individuals in Sao Paulo, Brazil, 2010. Cien Saude Colet 2014; 19(8):3327-3334.
33. Aguiar LB, Baccaro LF, de Souza Santos Machado V, Pinto-Neto AM, Costa-Paiva L. Disability and multimorbidity in women older than 50 years: a population-based household survey. Menopause 2015; 22(6):660-666.

34. Santos KA, Koszuoski R, Dias-da-Costa JS, Pattussi MP. Fatores associados com a incapacidade funcional em idosos do Município de Guatambu, Santa Catarina, Brasil. Cad Saude Publica 2007; 23(11):2781-2788.

35. Corona LP, Pereira de Brito TR, Nunes DP, da Silva Alexandre T, Ferreira Santos JL, de Oliveira Duarte YA, Lebrão ML. Nutritional status and risk for disability in instrumental activities of daily living in older Brazilians. Public Health Nutr 2014; 17(2):390-395.

36. Lima-Costa MF, Turci M, Macinko J. Saúde dos adultos em Belo Horizonte. Belo Horizonte: Núcleo de Estudos em Saúde Pública e Envelhecimento/Fiocruz, Universidade Federal de Minas Gerais; 2012.

37. Katz S, Ford AB, Moskowitz RW, Jackson BA, Jaffe MW. Studies of illness in the aged. The index of ADL: a standardized measure of biological and psychosocial function. JAMA 1963; 185(12):914-919.

38. Lawton MP, Brody EM. Assessment of older people: self-maintaining and instrumental activities of daily living. Gerontologist 1969; 9(3):179-186.

39. Webber SC, Porter MM, Menec VH. Mobility in older adults: a comprehensive framework. Gerontologist 2010; 50(4):443-450.

40. Newson RB. Attributable and unattributable risks and fractions and other scenario comparisons. Stata J 2013; 13(4):672-698.

41. Lima-Costa MF, Matos DL, Camargos VP, Macinko J. Tendências em dez anos das condições de saúde de idosos brasileiros: evidências da Pesquisa Nacional por Amostra de Domicílios (1998, 2003, 2008). Cien Saude Colet 2011; 16(9):3689-3696.

42. Parahyba MI, Simões CCS. A prevalência de incapacidade funcional em idosos no Brasil. Cien Saude Colet 2006; 11(4):967-974.

43. Hung WW, Ross JS, Boockvar KS, Siu AL. Association of chronic diseases and impairments with disability in older adults: a decade of change? Med Care 2012; 50(6):501-507.

44. Alexandre TS, Corona LP, Nunes DP, Santos JL, Duarte YA, Lebrão ML. Disability in instrumental activities of daily living among older adults: gender differences. Rev Saude Publica 2014;48(3):379-89.

45. Yokota RT, de Moura L, Andrade SS, de Sá NN, Nusselder WJ, Van Oyen H. Contribution of chronic conditions to gender disparities in disability in the older population in Brazil, 2013. Int J Public Health 2016; 61(9):1003-1012.

46. Adamson J, Beswik A, Ebrahim S. Is stroke the most common cause of disability? J Stroke Cerebrovasc Dis 2004; 13(4):171-177.

47. Skolarus LE, Burke JF, Brown DL, Freedman DA. Understanding stroke survivorship: expanding the concept of poststroke disability. Stroke 2014; 45(1):224230 . 
48. Dodson JA, Arnold SV, Reid KJ, Gill TM, Rich MW, Masoudi FA, Spertus JA, Krumholz HM, Alexander KP. Physical function and independence 1 year after myocardial infarction: observations from the Translational Research Investigating Underlying disparities in recovery from acute myocardial infarction: Patients' Health status registry. Am Heart J 2012; 163(5):790-796.

49. Van Jaarsveld CH, Sanderman R, Miedema I, Ranchor AV, Kempen GI. Changes in health-related quality of life in older patients with acute myocardial infarction or congestive heart failure: a prospective study. $J \mathrm{Am}$ Geriatr Soc 2001; 49(8):1052-1058.

50. Pinsky JL, Jette AM, Branch LG, Kannel WB, Feinleib M. The Framingham Disability Study: relationship of various coronary heart disease manifestations to disability in older persons living in the community. $\mathrm{Am} \mathrm{J}$ Public Health 1990; 80(11):1363-1367.

51. Gregg EW, Beckles GLA, Williamson DF, Leveille SG, Langlois JA, Engelgau MM, Narayan KM. Diabetes and physical disability among older U.S adults. Diabetes Care 2000; 23(9):1272-1277.

52. Kalyani RR, Saudek CD, Brancati FL, Selvin E. Association of diabetes, comorbidities and AlC with functional disability in older adults. Diabetes Care 2010; 33(5):1055-1060.

53. Maggi S, Noale M, Gallina P, Marzari C, Bianchi D, Limongi F, Crepaldi G. Physical disability among older Italians with diabetes. The ILSA Study. Diabetologia 2004; 47(11):1957-1962.

54. Nascimento CDEM, Mambrini JV, De Oliveira CM, Giacomin KC, Peixoto SV. Diabetes, hypertension and mobility among Brazilian older adults: findings from the Brazilian National Household Sample Survey (1998, 2003 and 2008). BMC Public Health 2015; 15:591.
55. Andrade FC, Guevara PE, Lebrão ML, Duarte YA. Correlates of the incidence of disability and mortality among older adult Brazilians with and without diabetes mellitus and stroke. BMC Public Health 2012; 12:361.

56. Alexandre Tda S, Corona LP, Nunes DP, Santos JL, Duarte YA, Lebrão ML. Gender differences in incidence and determinants of disability in activities of daily living among elderly individuals: SABE study. Arch Gerontol Geriatr 2012; 55(2):431-437.

57. Chrestani MAD, Santos IS, Matijasevich AM. Hipertensão arterial sistêmica auto-referida: validação diagnóstica em estudo de base populacional. Cad Saude Publica 2009; 25(11):2395-2406.

58. Selem SSC, Castro MA, César CLG, Marchioni DML, Fisberg RM. Validade da hipertensão autorreferida associa-se inversamente com escolaridade em brasileiros. Arq Bras Cardiol 2013; 100(1):52-59.

59. Oldridge NB; Stump TE. Heart disease, comorbidity, and activity limitation in community-dwelling elderly. Eur J Cardiovasc Prev Rehabil 2004; 11(5):427-434.

60. Medical Research Council Cognitive Function and Ageing Study (MRC CFAS), Resource Implications Study (RIS MRC CFAS). Profile of disability in elderly people: estimates from a longitudinal population study. BMJ 1999; 318(7191):1108-1111.

Artigo apresentado em 29/03/2017

Aprovado em 01/08/2017

Versão final apresentada em 03/08/2017 